\title{
Horizons/Théâtre
}

Revue d'études théâtrales

\section{Les traditions orales en Afrique : une exploration du conte comme source d'inspiration du théâtre moderne africain}

\section{Rodrigue Homero Saturnin Barbe}

\section{(2) OpenEdition}

Journals

Édition électronique

URL : https://journals.openedition.org/ht/1002

DOI : $10.4000 /$ ht. 1002

ISSN : 2678-5420

Éditeur

Presses universitaires de Bordeaux

Édition imprimée

Date de publication : 31 décembre 2018

Pagination : 54-67

ISBN : 979-10-300-0318-5

ISSN : 2261-4591

Référence électronique

Rodrigue Homero Saturnin Barbe, "Les traditions orales en Afrique : une exploration du conte comme source d'inspiration du théâtre moderne africain », Horizons/Théâtre [En ligne], 13 | 2018, mis en ligne le 01 janvier 2019, consulté le 17 mai 2022. URL : http://journals.openedition.org/ht/1002 ; DOI https://doi.org/10.4000/ht.1002

\section{(c) (i) $(9)$}

La revue Horizons/Théâtre est mise à disposition selon les termes de la Licence Creative Commons Attribution - Pas d'Utilisation Commerciale - Pas de Modification 4.0 International. 


\section{ROdRIGUE HOMERO SATURNIN BARBE}

Rodrigue Homero Saturnin Barbe est titulaire d'un Ph. D. en littérature et arts de la scène et de l'écran, concentration " études théâtrales ». II a effectué un stage postdoctoral à l'Université Laval (Québec) et sa recherche porte sur une étude comparative impliquant les formes de théâtre " anti-pouvoir » en Afrique et dans les Balkans, sous la direction de Liviu Dospinescu. Il a publié : « La littérature orale en Centrafricain et les nouvelles formes d'écritures » (Émergence, 2015, Tangence, Université de Trois-Rivières, Québec-Canada), "Les comédiennes en Centrafricain » (dans Théâtre au féminin, 2015, Africultures, Paris), "Le théâtre populaire en milieu urbain africain : dialogue ou antagonisme culturel entre tradition et modernité » (dans Culture and Litterature Studies, Annales Universitatis apulensis, séries philoloica, n 17, tome 2, 2016), "Le théâtre populaire en Afrique noire postcoloniale : mémoire, identité et révolution » (dans Simona Jisa, Buata B. Male et Sergio Miscoiu, Littérature et politique en Afrique, éd. Cerf Patrimoine, 2018).

Mail: barb_rodrigue@yahoo.fr

Résumé : Le conte paraît un genre littéraire impérissable. II a nourri l'imaginaire des sociétés à traditions orales pendant des millénaires et devient, de nos jours, l'une des disciplines littéraires qui ne cessent de capter l'intérêt des arts de la scène et de l'écran dans les sociétés dites " modernes". En Afrique, le conte fait encore partie des sources intarissables d'inspiration pour les arts du spectacle, notamment le théâtre. Pour une raison ou pour une autre, cet art de la parole se place au centre de l'éveil créateur de certains dramaturges et met-

Abstract : Storytelling seems to be an imperishable literary genre. It has nurtured the imagination of societies with oral traditions for thousands of years and is nowadays one of the literary disciplines which contihues to capture the activities of performing and screen arts in the so-called "modern societies". In Africa, storytelling is still one of the inexhaustible sources of inspiration for performing arts, especially theater. For some reason, this speech art is at the center of the creative awakening of some African teurs en scène africains. De là, on est en droit de s'interroger sur les motivations de ces artistes, ces créateurs qui restent attachés à l'oralité africaine. Dans cette étude, il s'agit d'examiner ce qui motive les créateurs dramatiques africains à accorder une place de choix à la tradition orale, notamment au conte pour rendre plus populaire leurs productions théâtrales.

Mots-cLÉs : conte, théâtre moderne africain, dramaturgie et mise en scène contemporaine, Afrique.

playwrights and directors. This enable us to wonder about the motivations of these artists, these creators who remain committed to African orality. In this study, we will examine what motivates African dramatic creators to give a place of importance to oral tradition, especially storytelling, in their work to make their theatrical productions more attractive. .

KeYwords : storytelling, modern African theater, dramaturgy and contemporary staging, Africa. 


\section{Les traditions orales en Afrique : une exploration du conte comme source $d$ 'inspiration du théâtre moderne africain}

\section{Introduction}

LE THÉÂTRE AFRICAIN MODERNE NE CESSE DE SE NOURRIR de l'oralité locale. Il est même difficile de remettre en question le rôle important joué par la littérature orale africaine, notamment le conte, l'épopée, le mvet ou les légendes dans l'émergence de la dramaturgie africaine moderne. Le dramaturge Gilbert Doho : «La dramaturgie camerounaise est redevable à notre littérature orale. D'ailleurs, pouvait-il en être autrement lorsque, dans quelques cieux que ce soient, l'oral précède toujours l'écrit ${ }^{1}$ ? » En effet, depuis les premières créations dramatiques africaines modernes, le conte traditionnel africain continue de se montrer particulièrement généreux pour le théâtre. S’il n'est pas la source directe d'une adaptation théâtrale dans certains cas, dans d'autres, il est omniprésent dans la structure d'une pièce où la présence d'un narrateur s'impose. Mais aussi, il n'est pas rare de voir toute une dramaturgie se construire à partir d'un récit ou une légende traditionnelle. Car le conte traditionnel africain se présente toujours comme le miroir de la société. Ses atouts artistiques, esthétiques et culturels ont suscité de capter les intérêts des arts du spectacle moderne en Afrique.

Particulièrement au cours de la décennie 1930, on peut dire avec assurance que le conte traditionnel s'affichait comme l'une des sources intarissables d'inspiration des premières pièces écrites. Selon les historiens, l'École William-Ponty sous l'impulsion de Charles Béart, alors directeur, encourageait les étudiants de son école à s'initier à l'écriture dramatique. Ainsi pour les recherches d'histoires ou de sujets, les étudiants étaient particulièrement orientés vers la collecte et l'exploration des contes, légendes, épopées, etc. $\mathrm{Au}$ bout du compte, l'histoire nous révèle que plusieurs dramaturgies africaines de souche (dans le sens propre du terme) sont nées de cette expérience scolaire $^{2}$. De même, entre 1960 et 1990, les artistes n’ont pas cessé de puiser dans le conte traditionnel. Mais aujourd'hui, des créations montrent que le 
conte attire encore des intérêts du théâtre moderne africain. Que recherchent les dramaturges et metteurs en scène africains dans les ressources du conte ? Que vaut le conte aujourd'hui dans l'enrichissement du théâtre lorsqu'on sait que l'Afrique, dans son ensemble, est ouverte au monde à travers les nouvelles technologies et que l'influence des nations culturellement puissantes engendre un étouffement considérable de la culture et de la civilisation africaine ?

Pour des besoins d'arguments concrets, il convient de clarifier nos idées en nous appuyant sur quelques exemples de contes populaires d'Afrique centrale, en particulier de Nnan Ndenn Bobo de René Philombe (1994).

\section{Regard bienveillant sur le conte africain}

Depuis les temps immémoriaux, le conte fait partie du patrimoine oral de l'humanité. Il se transmet (dans certaines sociétés encore) de génération en génération par la parole ${ }^{3}$ et rapporte à l'imaginaire collectif, à travers lequel se véhiculent les savoirs, les valeurs culturelles, la civilisation du peuple dont il est issu. Mais avant de mieux le valoriser, de vanter ses atouts, ses «vertus $\gg$ (parlant du conte), notamment pour éviter de tomber dans une absence d'érudition conceptuelle, il nous apparaît nécessaire de le définir et de le situer dans un contexte précis : africain. En effet, il n'est pas évident, jusqu'ici, d'observer beaucoup d'écart sur la question définitoire du mot « conte » dans le monde. Car il peut être admis comme le plus planétaire des genres littéraires et artistiques oraux. Toutefois, chaque société lui accorde une définition en lien avec ses propres valeurs, son histoire, son milieu et sa situation particulière, malgré le caractère universel du $\operatorname{mot}^{4}$. Pour la société occidentale par exemple, le conte se définit, à travers le dictionnaire Larousse, comme un récit, généralement assez court, des faits imaginaires. Dans un sens global, il est identifié à l'égal d'un récit ou d'une dramatisation qui engage scéniquement des personnages fictifs et allégoriques (humains, animaux, végétaux, objets ou les dieux) dont les aventures se situent dans un cadre irréel ${ }^{5}$. En Afrique, le conte, par sa nature et ses fonctions, est perçu, selon Marius Ano N'Guessan, comme « un récit oral, populaire [et vivant], traditionnel, à tendance ludique, didactique, magique, fictive ou réaliste, reflétant une certaine "vision du monde" de la communauté qui l'a produit ${ }^{6} \gg$. À cette définition de l'auteur ivoirien, ajoutons que le conte africain traditionnel se présente d'abord comme un miroir de la société, un « tout » artistique, culturel et social. En effet, le conte africain révèle dans sa forme traditionnelle un caractère rustique reflétant l'image d'une Afrique travailleuse, ingénieuse, imaginative, 
tolérante, généreuse, accueillante, vivante, festive et ouverte. Ces qualités dévoilées, à travers son contenu, font de lui un outil particulier de communication et de communion populaire, mais aussi un instrument d'éducation, de partage des valeurs de paix et d'harmonie.

Le conte africain traditionnel est également un jeu spectaculaire, ludique et un meilleur moyen pour les populations de croire en des rêves communs et individuels. En effet, chaque Africain qui reconnait encore au conte de tels potentiels sait pertinemment que s'épanouir au plan intellectuel, culturel, historique, social et spirituel à travers les mots du conteur, maître incontesté de la parole et «bibliothèque et archive vivante » d'une communauté, c'est se mettre à l'abri de l'ignorance, de la digression populaire. Amadou Hampaté Bâ disait ceci fièrement : «Je suis un diplômé de la grande université de la Parole enseignée à l'ombre des baobabs ${ }^{7}$. » Dans cet environnement où la tradition orale s'offre en une des sources capitales d'érudition pour un peuple, les moments de conte permettent d'écouter, de questionner, de consigner, de comparer, mais surtout de réfléchir (développer son esprit critique) aux idées émises dans un récit. Cela se présente comme dans un espace d'apprentissage conventionnel à l'occidentale, mais à la seule différence que, dans le contexte de l'oralité africaine, les prises de notes sont cérébrales. C'est dans cette école que le passé, le présent, et le futur de l’Afrique sont identifiés avec justesse et vérité. C'est pourquoi, dans son rôle artistique de reflet de la société, le conte agit dans la vision globale de contribuer à la compréhension des phénomènes existentiels complexes. Pourquoi l'Afrique est-elle un nid de crises chronique ? Où vont-ils, les Africains, avec les dieux d'autrui s'ils ne cessent eux-mêmes de « tuer » les leurs sans regret ? À ce genre de questionnement par exemple, le conte, les mythes, les légendes, les épopées interviennent en alternatives de réponses souvent très cohérentes. Cela exige donc du conteur une certaine prédisposition, une culture élevée dans l'originalité des fictions. Il s'agit d'inventer un récit avec une structure plausible, crédible, malgré le côté habituellement fantastique de la narration, mais tout (personnages, actions, péripéties, etc.) doit refléter les aspirations, les espérances de l'auditoire. Autrement dit, la narration nécessite des arguments, des commentaires, des réflexions, des techniques de persuasion à la hauteur des attentes populaires sur un sujet donné. Le conteur doit alors s'armer de beaucoup d'imagination et de créativité. C'est à partir de là que le conteur recourt aux proverbes, dictons, devinettes, expressions et autres rhétoriques de l'oralité afin de rendre son ou ses récits mémorables pour sa communauté. En effet, il faut inventer les personnages (humains, animaux ou végétaux, dieux, etc.), imaginer leurs actions, leurs fonctions, leur donner la parole et les mettre en 
scène dans un conflit irréel ou vraisemblable. Rappelons que le conte, en général, est moins long que le mythe, la légende ou l'épopée. Pour parler de tout un phénomène social ou culturel dans un temps court, cela exige un talent extrêmement élevé en matière de synthèse. De ce point de vue, le conte pourrait être comparé à une dramaturgie originelle. On peut même se permettre ici, sans exagération, de l'estimer comme un précieux pas vers la reconstitution du passé africain lointain (non consigné dans les archives officielles), un moyen de donner des explications à un présent complexe et d'inventer un futur solide pour la sauvegarde de la culture africaine.

De plus, en Afrique traditionnelle, une soirée de conte est généralement un moment de joie, de fête et de détente communautaire. À l'inverse des prestations familiales où seuls les parents racontent des récits pour participer à l'éveil d'esprit des jeunes (dans le cadre de l'éducation parentale), les activités engageant le conte sur la place du village (ou publique) donnent souvent lieu à un spectacle complet. Dans le cadre d'un événement populaire, on se raconte des histoires, on échange des proverbes, des devinettes et des expressions, mais le moment est aussi particulièrement festif. En effet, le conteur que nous considérons comme un acteur complet (au bon sens du terme), le «maitre du jeu communautaire, le « Joker » $\gg$ raconte des histoires, échange avec son auditoire pour le faire participer à la construction des pensées communes locales. Il chante des chansons populaires, danse et joue des instruments de musiques, ou bien il se fait accompagner par l'assistance qui collabore à l'ambiance de la soirée en apportant du rythme à travers, par exemple, les battements des mains.

Par ailleurs, le conte traditionnel en Afrique utilise généralement de l'affabulation au second degré pour s'exprimer. Dans la majorité des cas, rappelonsle, cet art de la parole est utilisé pour éveiller les consciences humaines. Autrement dit, rares sont les contes qui sont dits pour simplement plaire ou distraire. En fait, il n'est pas utilisé, comme on peut l'observer dans les sociétés occidentales, pour bercer et aider les enfants à trouver le sommeil. Il agit au-delà de ce besoin individuel. Chaque conte qui se dit en Afrique a pour nécessité de tenir en éveil les esprits ignorants, de conscientiser les imprudents, de dénoncer les comportements asociaux et injustes, de prévenir les malheurs communautaires et individuels, etc. C'est pourquoi le conte fait partie des fonds spectaculaires traditionnels qui s'expriment toujours de façon déguisée, en utilisant un monde fictif et un détour des événements ou des réalités de la société des hommes ${ }^{8}$. Il a cet avantage de parler des humains en se camouflant dans les univers zoomorphes. Il s'agit ici des animaux qui parlent, qui se montrent plus sensés, plus solidaires que les êtres humains. Ce 
détour renvoie toujours aux réalités, à la vie de tous les jours, au quotidien des Hommes. Si, par exemple, le lion est tourné en dérision par le singe, cela pourrait refléter, dans une certaine mesure, les relations entre les hommes de pouvoir (les puissants, les plus forts) et les petites gens. En matière de critique sociale et politique, le conte traditionnel africain est d'une inspiration remarquable lorsqu' il utilise un style imagé de dénonciation publique.

\section{Les rapports du conte avec le théâtre africain moderne}

On le sait, le théâtre est d'abord un art de spectacle vivant depuis la base. Même dans les plus anciennes formes de théâtre traditionnel africain, à l'exemple du Kotéba malien (qui a survécu à la modernité), il s'intéresse à l'humanité. Il parle de la vie humaine : les souffrances, la joie, la justice, l'injustice, la mort, l'amour, en suivant les traces du conte et les autres genres de l'oralité dramatique. Dans le cas du théâtre africain moderne dont la naissance (selon les historiens du théâtre africain) est située vers le début des années 1930 (nous l'avions évoqué plus haut dans la partie introductive de cette étude), le conte occupe une place prépondérante dans la genèse de la construction des idées dramaturgiques. Les historiens (à l'instar de Jacques Scherer, 1992) qui se sont penchés sur la question des origines du théâtre en Afrique ne cesser de l'affirmer : le théâtre puise beaucoup dans l'oralité, surtout dans le conte pour débuter ${ }^{9}$. Faisant partie des manifestations artistiques et culturelles traditionnelles prisées notamment de l'abondante littérature orale africaine, le conte a favorisé l'inspiration des premières dramaturgies et mises en scène du nouveau théâtre africain, et ce, depuis le contact des Africains avec la culture occidentale. De lui (du conte) seul, le théâtre pouvait recevoir le synopsis d'une histoire à raconter. Et comme le conte africain adore parler des humains sous les formes déguisées (en utilisant notamment la métaphore zoomorphique, les personnages esprits, des morts, des paroles tirées des proverbes, etc.), le nouveau théâtre africain moderne se voulait une signature particulière quant à la présentation de la vie africaine authentique et la valorisation de la culture africaine au monde extérieur. C'est de cette manière que le conte et les autres fonds spectaculaires traditionnels se faisaient une place de choix dans les tentatives d'initiation des élèves (Bernard Dadié, y compris) de l'École William-Ponty au théâtre de Molière, de Shakespeare ou d'Aristophane. Bakary Traoré (1958), cité par Rodrigue Homero Saturnin Barbe (2014), raconte que Charles Beart, alors directeur de l'école Ponty, pour initier les élèves de son établissement à l'écriture théâtrale, « a institué des "devoirs de vacances", dont les sujets portaient sur la vie et les coutumes 
indigènes ; au cours des vacances précédant la dernière et troisième année de scolarité, les élèves se documentaient sur le sujet choisi et présentaient le résultat de leurs études sous forme de mémoire $\gg^{10}$. Leurs sources de documentation n'étaient nulle autre qu'auprès des anciens, des conteurs, des griots, des vieillards, personnalités qui, naturellement, agissent en sages, en artistes dotés d'abondants talents reconnus dans leurs communautés respectives. Ceux-ci deviennent des bibliothèques, pour ne pas dire des archives artistiques vivantes. À ce propos, on peut se permettre de dire que les premières pièces écrites, issues de l'école Ponty, appartiennent au patrimoine populaire de toute l'Afrique noire, notamment francophone. Car, au cours de ces collectes de données, les soirées de contes traditionnels devenaient des objets d'observations intellectuelles, artistiques et esthétiques. Aucun droit d'auteur n'avait été réclamé. Dans ce contexte, les récits, les jeux et les démarches artistiques et esthétiques des conteurs agissaient ici en sources d'enrichissement de talents des jeunes créateurs de l'école Ponty.

De plus, les pratiques spectaculaires des rituels traditionnels qui traduisaient certaines émotions de la vie spirituelle, célébrant des héros du passé de l’Afrique, participaient également à la construction de la nouvelle création dramatique africaine.

De même que pendant la période postcoloniale, le conte africain ne cesse d'inspirer le théâtre moderne. Il se constituait en une des richesses artistiques, en ce qui a trait à la mobilisation populaire, à la conscientisation et à la distraction du spectateur. Soulignons d'ailleurs que la portée historique, sociale et culturelle des contes est considérable en Afrique. Son exploitation au théâtre postcolonial a offert aux dramaturges et metteurs en scène des techniques de présentation des hauts faits ancestraux. Pour de nombreux artistes africains postcoloniaux, le conte regorge d'outils très efficaces favorables au soutien du théâtre moderne, dans sa perpétuelle quête de stimulation des prises de conscience collective et individuelle sur les problèmes qui nuisent à l'émancipation de l'Afrique. Lorsqu'il inspire le théâtre, c'est souvent pour le nourrir efficacement à faire adopter aux spectateurs, membres d'une communauté, des solutions à l'image des valeurs propres et communes à l'Afrique ${ }^{11}$.

Enfin pour certains dramaturges des années 1960 à 1990, le théâtre africain moderne a besoin de l'oralité, notamment du conte pour conserver l'identité africaine authentique dans la composition d'une pièce de théâtre. Cela permet en effet de réduire les écrasantes influences étrangères dans les conceptions dramaturgiques et de favoriser la perpétuation de la culture africaine. Dans ce sens, au lieu que William Shakespeare ou Molière soit le premier et 
la seule référence en matière d'inspiration pour le classique africain, le conte se présenterait comme alternative raisonnable. Il peut aider ici au brassage culturel dans le but d'atténuer le sentiment de déculturation de l'Afrique à travers son théâtre moderne, du moment où celui-ci évolue dans un environnement citadin, le plus souvent. De plus, placer le conte parmi les centres d'intérêt de la création théâtrale postcoloniale a permis également aux créateurs de faire redécouvrir les traditions locales aux populations des villes africaines en proie à la vision du monde extérieur.

\section{Étude de cas de Nnan Ndenn Bobo de René Philombo et autres contes populaires centrafricains}

Dans cette partie de notre réflexion, nous souhaitons observer quelques éléments attractifs qui continuent de placer le conte comme l'un des favoris du théâtre moderne africain en matière d'inspiration. Cette observation se fera en s'appuyant sur des contes populaires de l'Afrique centrale, notamment chez les peuples bantous. Nnan Ndenn Bobo de René Philombe, un texte écrit en 1994, sera notre principal support d'analyse. Pour commencer, qu'est-ce qui pourrait instituer Nnan Ndenn Bobo en un conte inspirant pour la dramaturgie contemporaine en Afrique ? Pour dégager quelques éléments de réponses à cette question, nous souhaitons nous appuyer sur quelques aspects : la thématique, la structure, le style du récit et la culture. Mais avant de les explorer, il nous paraît nécessaire de présenter brièvement le contenu du récit de René Philombe. Nnan Ndenn Bobo est un conte traditionnel béti, un peuple de la forêt vivant au centre et au sud du Cameroun. Il raconte l'histoire du Ndenn Bobo, un personnage lugubre, un sociopathe, un méchant (à l'image du diable, du point de vue des habitants du Ciel et de la Terre), mais qui est élevé, sinon nommé, au rang de conseiller et favori de Zama (Dieu). Le récit finit dans une accusation, un procès absurde que Ndenn Bobo engage contre Zama, l'accusant de «l'Être le plus méchant de l'univers », un être qui mérite la plus terrible des sentences, selon Zama lui-même. Autrement vu, Lucifer traduit Dieu en justice en démontrant qu'il est le monstre de l'injustice dans tout l'univers.

Nnan Ndenn Bobo aborde une thématique très politique. En effet, lorsque le conte africain s'attaque à la question de justice, cela inclut naturellement le problème du pouvoir et de la gestion de la cité. Le théâtre grec antique, par exemple, s'est fait remarquer par son intérêt de rendre l'art théâtral public, le mettant au service de l'État. À ce titre, les dramaturges se déployaient pour traduire la vie publique à travers la tragédie, d'une part. D'autre part, 
ceci permet d'entendre la voix des petites gens grâce à la comédie. En lançant un regard rapide sur les origines de ce théâtre occidental, il n'est pas rare que les mythes, le conte et les légendes se constituent en source d'inspiration ${ }^{12}$. Nnan Ndenn Bobo a les mêmes atouts que les mythes et les épopées grecs, arts originellement oratoires. Il aborde la question qui touche la gestion du pouvoir, la justice de Dieu. Le récit parle d'un conflit entre Dieu (Zama) et son protégé (Ndenn Bobo). Mais lorsque l'on porte une attention de second degré à ce récit cocasse, on est loin de se tromper lorsque l'on admet que l'auteur se montre moins tendre, très critique, très satirique vis-à-vis de certains pouvoirs autocratiques en Afrique. Car, à bien y voir, le conflit entre Zama et Ndenn Bobo dévoile, de façon allégorique, les relations entre les hommes de pouvoir africains et leurs peuples, en l'occurrence les opposants. Dans ce contexte, on peut se permettre de dire ici que René Philombe nous présente la tragédie dans laquelle l'Afrique subit la justice des hommes de pouvoir immatures. Les actes, gestes et décisions du président ou du roi sont « naturellement justes $\gg$. Ici, il est impossible de remettre en question la justice du « Père de la nation », du « Guide », du « Roi », etc. Se trompe-t-il parfois ? A-t-il toujours un bon jugement ? Toutes ces questions non exhaustives permettent au théâtre de puiser des arguments conséquents contribuant à traiter, avec efficacité, les questions du pouvoir et de la vraie justice en Afrique. Car Nnan Ndenn Bobo montre, à travers sa thématique, qu'il est doté de tout ce qui est utile pour que le théâtre moderne africain puisse profiter des atouts satiriques d'un conte dans son rôle de miroir social. Il s'exprime, de façon déguisée, en réaction contre les pouvoirs absolus et les violences inhérentes à ceux-ci. On peut voir aussi que René Philombe cherche à se hisser, à travers ce récit, à la hauteur des effroyables tragédies nées des dictatures dans les pays africains. En fait, ce conte utilise de la satire pour dénoncer le désordre créé par les mauvaises décisions de Zama (le Guide suprême) et fait de l'humour son arme de combat contre la violence que subit le peuple (les Africains) à cause des abus de pouvoir.

En ce qui concerne la structure, l'auteur de Nnan Ndenn Bobo organise le conte en séparant les événements de sorte qu'il y ait une exposition, un conflit, des péripéties et un dénouement. Dans le texte de René Philombe, l'exposition présente l'environnement dans lequel les Bantoues vivaient. « Il $\mathrm{y}$ a longtemps, très longtemps les habitants du Ciel et de la Terre vivaient en harmonie sous la direction de Zama Élopogo... » Cette partie introductive du conte servirait bien d'inspiration pour une dramaturgie engagée. Elle présente ici une société harmonieuse au départ. Mais la suite risque d'être autrement. En effet, un conflit naîtra au moment où Zama décide de nommer 
Ndenn Bobo (un homme méchant, asocial, etc.) au cœur de son pouvoir en tant que conseiller et favori. Comment un Dieu si bon, si juste et si parfait pouvait-il s'entourer d'une personne aussi singulièrement lugubre (à l'image du diable) pour collaborer dans la gestion, les prises de décision des choses publiques?

Les péripéties dans ce conte nous renvoient aux comportements « dithyrambiques » de Ndenn Bobo face aux désirs et aux pouvoirs de Zama. Par exemple, à la fête des récoltes, « tout le monde est convié au palais de Zama Élopogo, tout le monde doit être présent sans exception. Mais Ndenn Bobo était absent $\gg$. De quel droit se permet-il cela ? Ce personnage de Ndenn Bobo ne laissera pas indifférents certains dramaturges africains en quête d'un « citoyen-opposant et rebelle ». C'est un rôle idéal, dans une dramaturgie engagée, pour créer l'illusion d'un opposant dans un environnement de pouvoir autocratique contemporain en Afrique.

En ce qui a trait au style, l'auteur met en évidence la force de la métaphore. Ici, on parle des habitants du Ciel et de la Terre, des oiseaux de fer, de «Dieu » qui nomme « Lucifer » comme favori et conseiller, etc. Ce sont là des réalités absurdes pour un commun de mortel, pour Monsieur et Madame Tout-le-Monde. C'est dans cela que le dramaturge puiserait son style, ses ressources pour contourner efficacement les censures, s'il tient à parler des réalités sans s'exposer facilement aux réactions violentes des hommes de pouvoirs autoritaires et gérontocratiques en Afrique.

Enfin, l'identité culturelle africaine profonde réside encore dans les contes, mythes et épopées. Les valeurs universelles telles que le travail, le partage, le respect, l'esprit de fête, etc. sont des aspects essentiels que l'auteur de Nnan Ndenn Bobo tient à mettre en évidence. À une époque où le monde est devenu un petit village, le théâtre se doit d'exposer la culture africaine à travers la création. L'Afrique a les mêmes problèmes que les autres continents. Aujourd'hui, la culture africaine fait moins le poids aux regards des Africains eux-mêmes au profit des valeurs culturelles venant d'ailleurs. Or, dans le conte, on trouve régulièrement cette sociabilité africaine, la « grande famille africaine » qui pourrait inspirer les cultures occidentales, surtout, dans le sens de repenser les valeurs individualistes, qui, parfois, sont à l'origine de la fragmentation des contacts humains. En effet, l'auteur dit dans son exposition ceci : « Car grande était la générosité de Zama Élopogo. Grandes ouvertes étaient à tout moment les portes de l'immense palais de Zama Élopogo, où sans distinction de races, de tribus, ni de classes, de continent, de conditions. Tous les êtres se rendaient en masse pour fumer la vie, touiitouiiitouii, comme on fume chez 
les Turcs jusqu'à la béatitude ${ }^{13}$. $\gg$ À lui seul, cet extrait de Nnan Ndenn Bobo pourrait offrir à la création théâtrale un moyen de contribuer au rafraîchissement de la culture africaine et mondiale pour une humanité meilleure.

\section{Quel avenir pour le conte dans la création théâtrale contemporaine en Afrique?}

Il est difficile de répondre à cette question dans un contexte où l'art en général et le théâtre en particulier peinent à obtenir le soutien nécessaire des pouvoirs publics et du secteur privé pour leur épanouissement, mais aussi où le conte fait face à une ignorance de ses atouts chez les jeunes dramaturges africains. Pour la plupart des pays africains, les artistes (bourrés de talents) comptent souvent sur le soutien des structures étrangères. Au pire, ils utilisent leurs propres ressources financières, notamment, pour produire le théâtre. Et Dieu seul sait combien ces créateurs se battent pour que la culture africaine se taille une place respectable dans ce siècle du donner et du recevoir. Il devient donc difficile d'être optimiste quant à l'avenir commun du conte et du théâtre moderne africain. Qu'à cela ne tienne, l'espoir est toujours présent dans ces moments difficiles de la production théâtrale africaine qui manque d'attachement aux fonds spectaculaires traditionnels.

Au début des années 1960, Amadou Hampaté Bâ se battait pour que l'oralité africaine soit placée comme un des patrimoines immatériels mondiaux de l'humanité protégés par l'UNESCO. Ses démarches n'ont pas été suffisamment concluantes. Mais au moins, des moyens ont été accordés à une équipe qu'Hampaté Bâ dirigeait pour sauver quelques richesses orales, en voie de disparition, qui restaient en Afrique de l'Ouest, notamment. C'est dans ces circonstances que cet auteur malien de plusieurs livres de contes et ouvrages critiques portant sur l'oralité en Afrique de l'Ouest disait : « En Afrique quand vieillard meurt, c'est une bibliothèque qui brûle. »Ce cri de détresse n'a pas laissé indifférents certains créateurs des trois décennies postcoloniales. En effet, les théâtres de Were Were Liking, de Bernard Dadié, de Koulsy Lamko, de Guy Menga, de Sylvain Bemba pour ne citer que ceuxlà, ont reconnu les richesses artistiques, esthétiques et culturelles de l'oralité populaire africaine. À travers leurs pratiques (ou créations), il n'est pas rare d'observer l'utilisation (avec beaucoup de talent) des rituels, des contes, des mythes, etc. comme des ressources créatrices en vue d'élever le théâtre moderne africain. C'est la raison pour laquelle les historiens et les critiques ne manquent pas de référer à la plupart de ces personnalités pour situer la particularité de l'Afrique à travers son théâtre. 
Aujourd'hui, on s'interroge sur place du conte comme outil d'inspiration pour les dramaturges et metteurs en scène africains. Que peut-on encore tirer du conte pour rendre le théâtre plus attrayant tout en protégeant la culture africaine ? Pour que le théâtre joue pleinement son rôle dans cette « jungle » d'échange et de partage des cultures, les créateurs, selon leurs styles, doivent plus que jamais mettre à profit les atouts artistiques, esthétiques et culturels du conte afin de mieux négocier la place de l'Afrique dans les évolutions vertigineuses des nouvelles technologies. En effet, le fait de revisiter le conte, de l'investir, de l'impliquer dans les démarches de la création théâtrale contemporaine constitue une des meilleures façons d'archiver, de remémorer, de conserver la culture orale africaine sous une autre forme. Car aujourd'hui, les dramaturgies africaines sont facilement publiées, d'un côté. Avec les nouvelles technologies, de l'autre, les représentations sont filmées, archivées sur internet.

Le théâtre moderne africain conservera son originalité artistique, esthétique et culturelle que dans les intérêts qu'il continuera de porter aux atouts créateurs du conte. En effet, il apparait de plus en plus difficile que ce théâtre bénéficie de la bienveillance, de l'adhésion du grand public africain. Pour cause, les spectacles sont conçus davantage dans un modèle étranger aux réalités, aux besoins sociaux, politiques et culturels des populations locales. Le théâtre moderne africain donne l'impression de faire de l'art pour l'art, singeant relativement mal le style occidental. Or, ce n'est un secret pour personne, l'Afrique ne cesse d'être le théâtre de tous les mots de la planète. C'est justement le moment pour de l'art dramatique de jouer pleinement son rôle (suivant l'exemple des aînés tels que Bernard Dadié, Were Were Liking, et bien d'autres) dans le changement et la recherche des solutions aux multiples crises que connait le continent. Tout est dans la tradition orale africaine pour contribuer aux issues de sorties des faiblesses de l'Afrique. Alors, si l'on considère que rien n'est gratuit dans l'art africain, le conte ne tarit pas de ressources pour enrichir le théâtre actuel et du futur. Avec le conte, l'inspiration du théâtre moderne africain s'avoue infinie. Le conte, pris comme miroir de la société, est capable de traiter tous les sujets existentiels et même au-delà. Il parle des puissants, des faibles, aussi à la fois des personnes intelligentes et idiotes, des riches et des pauvres. Bref. Rien ou presque qui touche à la vie ne peut échapper à l'exploration du conte en Afrique. Avec lui, le théâtre moderne africain a tout pour inspirer (aussi) le théâtre mondial à travers, entre autres, les techniques élevées d'utilisation de la métaphore. Ici, le théâtre peut dénoncer sans désigner, sans provoquer sur les artistes la foudre de la censure, surtout les représailles, la colère des mécontents. Donc, en recourant au 
conte pour sa pratique théâtrale, le créateur contemporain a l'assurance de puiser son inspiration dans la tradition populaire, une tradition qui favorise le développement de la sagesse humaine, une sagesse où les héros, la vie, la philosophie et les rêves africains demeurent authentiques à la culture et la vision du monde de l'Afrique. Il s'enracine, de ce fait, dans une culture bien maîtrisée avant de s'approprier d'autres ressources tirées des ouvertures au monde. Avec une telle démarche, dramaturges et metteurs en scène favorisent la survie de la civilisation et des valeurs culturelles africaines, mais aussi permettront le renouvellement des techniques du théâtre moderne contemporain.

Enfin, l'exploitation du conte au théâtre pourrait aider à ranimer, à la lumière des nécessités contemporaines africaines, à brasser et à remettre en question certaines coutumes et traditions rétrogrades. En effet, si à travers les contes et légendes la société traditionnelle africaine est présentée fondamentalement gérontocratique, dans laquelle, par exemple, le culte des ancêtres et des morts, celui des génies tutélaires et une idéologie communautaire s'imposent, le théâtre moderne a besoin de ces fonds spectaculaires traditionnels pour renouveler, avec intelligence (et donc critique), les valeurs de l'Afrique aux idées universelles.

\section{Conclusion}

En somme, cette réflexion nous permet d'exposer quelques pistes aidant (nous l'espérons) le théâtre africain moderne à se réaffirmer localement, mais aussi à œuvrer efficacement dans l'optique de redorer l'image de la culture africaine, dans cet univers où chaque peuple lutte pour que sa vision du monde devienne la seule, celle qui est supérieure et valable pour toute l'humanité. Il serait prétentieux de penser que seul le conte porte des outils esthétiques, artistiques et culturels pour l'enrichissement du théâtre moderne africain. Loin de là. Toutefois, il est encore temps de réitérer les arguments qui encourageaient les dramaturges et les metteurs en scène africains, des années 1960 à 1990, à s'appuyer aussi sur le conte pour faire du théâtre un véritable miroir de la société africaine. Le théâtre moderne africain à venir manquera son renouvellement créateur, il sera absent au combat des nations en quête d'une culture forte (une culture à laquelle le monde entier revendique l'appartenance : la paix, l'humanité), s'il ignore totalement les potentiels de l'art oratoire africain au bénéfice de «l'évolution » technologique. En explorant le conte sous toutes les formes (adaptation, inspiration, etc.), le théâtre moderne africain poursuit l'inventaire de ses atouts, et ceci permet d'assurer la pérennité des sources intarissables d'inspiration au futur théâtre 
d'Afrique. Car les contes et les légendes sont comme un repère pour retracer, interroger le passé. Ils permettent au théâtre de recréer un « modèle » pour les jeunes générations du continent qui manquent cruellement de repères culturels, religieux et intellectuels locaux.

\section{Notes}

1. Gilbert Doho, « La dette du théâtre camerounais moderne envers l'oralité ", dans Bole Butake et Gilbert Doho (dir.), Théâtre camerounais, Yaoundé, Actes du colloque de Yaoundé, 1988, p. 67.

2. Charles Béart, "Le théâtre indigène et la culture franco-africaine ", L'éducation francoafricaine. Bulletin de l'enseignement de l'AOF, 1937, p. 3-4.

3. Catherine d'Humières, D'un conte à l'autre, d'une génération à l'autre, ClermontFerrand, Presses universitaires Blaise Pascal, 2008, p. 8-9.

4. Pierre N'da K., Le conte africain et l'éducation, Paris, L'Harmattan, 1984, p. 13.

5. Effoh Clément Ehora, Roman africain et esthétique du conte, Paris, L'Harmattan, 2013, p. 18.

6. Marius N'Guessan Ano, "Le conte traditionnel oral ", dans Notre Librairie, n 86, p. 38-39.

7. Bernard Magnier, Amadou Hampaté Bâ. Sur les traces d'Amkoullel l'enfant peul, Arles, Actes Sud, 1998, p. 11.

8. Idem, p. 16 .

9. Jacques Scherer, Le théâtre en Afrique noire francophone, Paris, Presses Universitaires de France, 1992, p. 22.

10. Bakary Traoré, Le théâtre négro-africain et ses fonctions sociales, Paris, Présence Africaine, 1958, p. 11, cité par Saturnin Homero Rodrigue Barbe dans «Le théâtre d'intervention en zone urbaine centrafricaine : rapports aux réalités quotidiennes et aux valeurs culturelles locales », thèse de doctorat, juillet 2014, Université Laval, Québec (QC) Canada, p. 35.

11. Bakary Traoré, «Le théâtre africain : réalités et perspectives », dans Le théâtre négro-africain, Actes du colloque d'Abidjan - 1970, Paris, Présence Africaine, 1970, p. 55.

12. Sylvie Chalaye, Nouvelles dramaturgies d'Afrique noire francophone, Rennes, Presses universitaires de Rennes, 2004, p. 130-131.

13. René Philombe, Nnan Ndenn Bobo, Yaoundé, Édition du CRAC, 1994, p. 7. 